ACCepted for publichtion in the Astrophysical Journal Letters

Preprint typeset using $\mathrm{AT}_{\mathrm{E} X}$ style emulateapj v. 08/22/09

\title{
TROUBLE FOR AGN FEEDBACK ? THE PUZZLE OF THE CORE OF THE GALAXY CLUSTER AWM 4
}

\author{
Fabio Gastaldello $^{1,2}$, David A. Buote ${ }^{1}$, Fabrizio Brighenti $^{2,3}$ \& William G. Mathews ${ }^{3}$, \\ Accepted for publication in the Astrophysical Journal Letters
}

\begin{abstract}
The core of the relaxed cluster AWM 4 is characterized by a unique combination of properties which defy a popular scenario for AGN heating of cluster cores. A flat inner temperature profile is indicative of a past, major heating episode which completely erased the cool core, as testified by the high central cooling time ( $\gtrsim 3 \mathrm{Gyr})$ and by the high central entropy level $\left(\sim 60 \mathrm{keV} \mathrm{cm}{ }^{2}\right)$. Yet the presence of a $1.4 \mathrm{GHz}$ active central radio galaxy with extended radio lobes out to $100 \mathrm{kpc}$, reveals recent feeding of the central massive black hole. A system like AWM 4 should have no radio emission at all if only feedback from the cooling hot gas regulates the AGN activity.

Subject headings: cooling flows - galaxies: clusters: general — galaxies: clusters: individual (AWM 4) - X-rays: galaxies: clusters
\end{abstract}

\section{INTRODUCTION}

The cores of galaxy clusters have been a long standing puzzle since the early X-ray observations. Initially gas in the cores of clusters was thought to cool, condense, and flow toward the center, as long as the cooling time was less than the age of the universe. Most $(\sim 2 / 3)$ galaxy clusters in the local universe satisfy this condition, with early estimates of their accretion rates as high as $10^{2}-10^{3}$ $M_{\odot} \mathrm{yr}^{-1}$. The problem with this interpretation was that the mass sink for all this supposedly cooling and condensing gas has never been found (the classical cooling flow problem or "mass-sink problem", e.g. Donahue \& Voit 2004). X-ray observations with Chandra and XMM have established that there is little evidence for emission from gas cooling below $\sim T_{\text {vir }} / 3$ (the new "spectroscopic $\dot{M}$ problem", Donahue \& Voit 2004). Just when gas should be cooling most rapidly it appears not to be cooling at all (e.g, Peterson \& Fabian 2006).

A compensating heat source must therefore resupply the radiative losses and many possibilities have been proposed, including thermal conduction (e.g., Narayan \& Medvedev 2001), energy released by mergers (e.g., Motl et al. 2004) and heating by an active AGN (e.g., Tabor \& Binnev 1993). AGN feedback heating has become the most appealing solution to the problem (for a recent and detailed review see McNamara \& Nulsen 2007) for the following reasons: 1) the high incidence of clusters with short central cooling times means they cannot be a transient phenomenon, requiring a heating rate which closely matches the cooling rate. This is difficult to explain, unless heating rates are coupled to cooling rates. AGN feedback is a natural vehicle to provide the coupling if the AGN is fed by cooled or cooling gas (Churazov et al. 2002); 2) there is clear observational evidence for AGN heating in cool core clusters, since a majority $(\sim 71 \%)$ harbour radio sources

\footnotetext{
${ }^{1}$ Department of Physics and Astronomy, University of California at Irvine, 4129 Frederick Reines Hall, Irvine, CA 92697-4575

2 Dipartimento di Astronomia, Università di Bologna, via Ranzani 1, Bologna 40127, Italy

${ }^{3}$ UCO/Lick Observatory, University of California at Santa Cruz, 1156 High Street, Santa Cruz, CA 95064
}

(Burns 1990). Following the launch of Chandra, "cavities" in the ICM of a similar fraction of cool core clusters (e.g., Dunn \& Fabian 2006) have been found which correlate with the radio emission of the central AGN. These have been interpreted as bubbles of relativistic plasma inflated by the radio jets causing $P d V$ heating (Churazov et al. 2002). Together with weak shocks associated with the outburst, long expected in models of jet-fed radio lobes (Scheuer 1974) and finally detected in deep Chandra observations, as for example, in M87 (Forman et al. 2005), Hydra A (Nulsen et al. 2005) and MS 0735+7241 (McNamara et al. 2005), the energies provided by the AGN are not only comparable to those needed to stop gas from cooling, but the mean powers of the outbursts are well correlated with the powers radiated (Nulsen et al. 2006); 3) AGN feedback has broader astrophysical implications for galaxy formation, explaining the truncation of the high end of the galaxy luminosity function and the symbiosis of black holes and spheroids, forming an impressive coherent picture. But we are far from a clear understanding, since "some loose ends remain" (Binney 2005).

Entropy can offer more direct insights into the processes that add and remove thermal energy from the gas. Motivated by a study of entropy profiles in a sample of cool core clusters (Donahue et al. 2006), Voit \& Donahue (2005) proposed a framework for AGN heating in line with the features discussed above and able to explain the observed core entropy profiles with episodic outbursts of $\sim 10^{45} \mathrm{erg} \mathrm{s}^{-1}$ occurring on a $\sim 10^{8}$ yr timescale. Stronger outbursts like those seen in Hydra A and MS 0735+7241, which are rarer and longer lasting may also play a role in extending the time between outbursts and in elevating the intra-cluster entropy beyond the core region of clusters. Another clue comes from the Chandra observations of two radio-quiet (at 1.4 GHz in the NVSS, Condon et al. 1998) clusters by Donahue et al. (2005): A 1650 and A 2240. Both clusters were classified as strong cooling flows by Peres et al. $(1998)$, but their central cooling times $\left(\sim 10^{9} \mathrm{yr}\right)$ are much longer than the central cooling times of cooling flow clusters with current radio activity $\left(\sim 10^{8} \mathrm{yr}\right)$; they 
also have flatter core entropy profiles with larger values of central entropy $\left(30-40 \mathrm{keV} \mathrm{cm}^{2}\right)$ compared to ones $(\sim 10$ $\mathrm{keV} \mathrm{cm}^{2}$ ) of the sample with active radio central galaxies. Either the gas in these clusters experienced such a dramatic episode of feedback heating sometime in the past that it has not required any additional feedback for $10^{9} \mathrm{yr}$ or mergers have kept the gas from cooling and condensing appreciably since the central galaxies were formed, as for example shown in the simulations by Burns et al. (2007). The fact that signs of AGN feedback appear only in those cool cores that currently require feedback has been interpreted as strengthening the case for AGN heating. In this scenario there is a clear dichotomy between active and radio quiet clusters: one would expect the cluster population to bifurcate into systems with strong temperature gradients and feedback and those without either (Donahue et al. 2005).

A different scenario for another radio quiet cluster, A 644, in the sample of Peres et al. (1998) was proposed by Buote et al. (2005). In that paper a merger scenario was proposed to explain the properties of the radio-quiet clusters A 644 and A 2589, which have almost isothermal temperature profiles and abundance profiles that decrease at large radii. Buote et al. (2005) suggested that the center offsets observed in these clusters $(\approx 60 \mathrm{kpc}$ A $644 ; \approx 10 \mathrm{kpc}$ A2589) indicated that they are settling down from a past merger event. The larger offset and central ( $\lesssim 50 \mathrm{kpc}$ ) disturbance in A644 indicates it is at an earlier stage after the merging. The cluster A2029 could represent an interesting later stage for these objects. Since A2029 has a cool core, a very small center offset $(\approx 4 \mathrm{kpc}$, smaller than A644 and A2589), with a WAT, yet its image morphology is otherwise highly relaxed, cooling has advanced further and the feedback episode has just started. It is interesting to note that of the objects in Peres et al. (1998) as listed in Donahue et al. (2005) lacking either an emission line nebula or a strong radio source, two without emission lines and radio activity (A 644 and A 1689, for the latter see e.g., Girardi et al. 1997) and one with emission lines but a weak radio source (A 2142, Markevitch et al. 2000) show signs of merger activity.

In this paper we present the properties of the low-mass cluster AWM4 at $z=0.0317$ which is a clear counterexample to the dichotomy proposed by Donahue et al. (2005). All distance-dependent quantities have been computed assuming $H_{0}=70 \mathrm{~km} \mathrm{~s}^{-1} \mathrm{Mpc}^{-1}, \Omega_{\mathrm{m}}=0.3$ and $\Omega_{\Lambda}=0.7$. At the redshift of $z=0.03171^{\prime}$ corresponds to $38 \mathrm{kpc}$. All the errors quoted are at the $68 \%$ confidence limit.

\section{THE PROPERTIES OF AWM 4}

AWM 4 is a poor cluster whose X-ray emission is extended and regular (see Fig.1) and the peak of the X-ray emission is coincident with the dominant giant elliptical NGC 6051. It has a bolometric luminosity (0.1-100 keV) of $3.93 \pm 0.06 \times 10^{43} \mathrm{erg} \mathrm{s}^{-1}$ and a mass weighted temperature of $2.48 \pm 0.06 \mathrm{keV}$ within $455 \mathrm{kpc}$, and a mass of $1.04 \pm 0.10 \times 10^{14} \mathrm{M}_{\odot}$ within $r_{500}=708 \pm 23 \mathrm{kpc}$. It therefore lies at the low end of the mass and temperature range defining clusters of galaxies. 28 galaxy members have been identified, most are absorption line systems with a strong concentration of early-type galaxies in the center and with a smooth gaussian velocity distribution

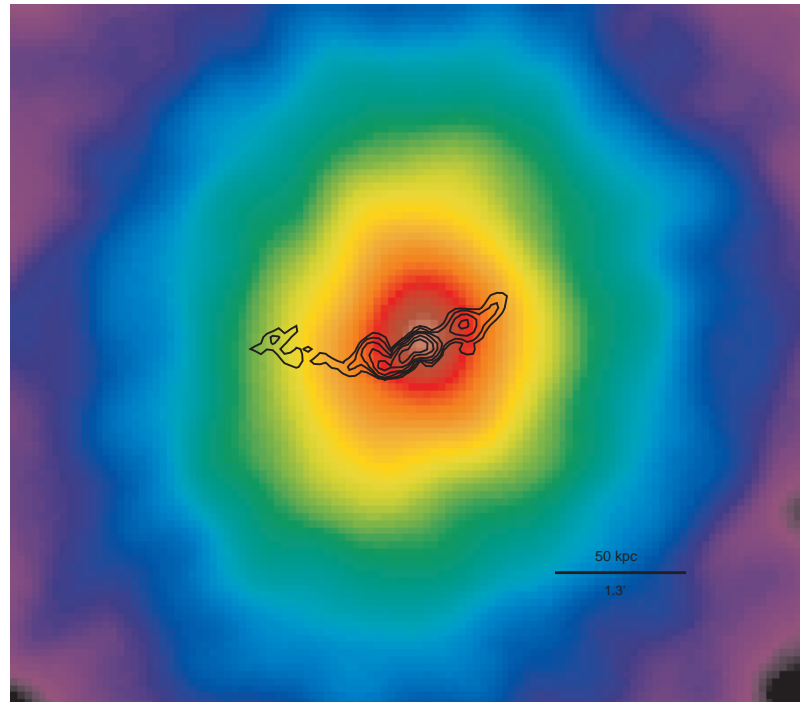

FIG. 1.- XMM 0.5-2 keV X-ray image of the core of the cluster AWM 4. The image combines data from the two MOS cameras: the image has been processed to remove point sources, flat fielded with a $1.25 \mathrm{keV}$ exposure map and smoothed with a $16^{\prime \prime}$ gaussian. Radio contours taken from the VLA FIRST $20 \mathrm{~cm}$ survey are superimposed in black.

centered at the velocity of NGC 6051; the velocity dispersion of the system is $439_{-58}^{+93} \mathrm{~km} \mathrm{~s}^{-1}$ (Koranyi \& Geller 2002). NGC 6051 is considerably brighter than the galaxies around it and recent deep $R$-band imaging (Zibetti et al., in preparation) has established the fossil nature of this system, according to the definition of Jones et al. (2003). The $X M M$ data (see Fig,2, upper right panel) show a clear abundance gradient, from $\sim 0.2 Z_{\odot}$ (in the units of Grevesse \& Sauval 1998) at $400 \mathrm{kpc}$ to $1.2 \pm 0.1$ in the inner $20 \mathrm{kpc}$, another indication of a fairly relaxed system (e.g., De Grandi et al. 2004). NGC 6051 harbors a powerful radio source with $1.4 \mathrm{GHz}$ flux of $607 \pm 22$ mJy (from the NVSS catalog, Condon et al. 1998) and spectral index $-0.72 \pm 0.04$ (calculated using radio fluxes from $26.3 \mathrm{MHz}$ to $10.55 \mathrm{GHz}$ taken from NED and from Neumann et al. 1994) corresponding to a total radio luminosity of $8.9 \times 10^{40} \mathrm{erg} \mathrm{s}^{-1}$ in the energy band $10 \mathrm{MHz}$ - $10 \mathrm{GHz}$. Radio contours taken from the FIRST $1.4 \mathrm{GHz}$ radio image (White et al. 1997) are also shown in Fig.1.

Given its relaxed appearance both in the X-ray and in the optical we included the object in our sample of 16 bright relaxed groups/poor clusters observed by either Chandra or XMM and chosen to be among the candidates best-suited for the application of the hydrostatic equilibrium approximation to measure their mass profiles (Gastaldello et al. 2007), where the details of the analysis of the available $X M M$ observation of AWM 4 can be found. The $X M M$ data show a unique temperature profile (see Fig[2, upper left panel) for a relaxed object, with an isothermal core out to $200 \mathrm{kpc}$ (as found also in O'Sullivan et al. 2005) and then a decline at large radii.

To characterize quantitatively the entropy profile, determined by computing the adiabatic constant $K=$ $k T n_{e}^{-2 / 3}$ (see Fig,2, lower left panel), we follow Donahue et al. (2006) and fit both a simple power law of the form $K=K_{100}(r / 100 \mathrm{kpc})^{\alpha}$ and the same power law plus a central constant $K=K_{0}+K_{100}(r / 100 \mathrm{kpc})^{\alpha}$. We find $K_{100}=184 \pm 25 \mathrm{keV} \mathrm{cm}{ }^{2}$ and $\alpha=0.71 \pm 0.03$ with 

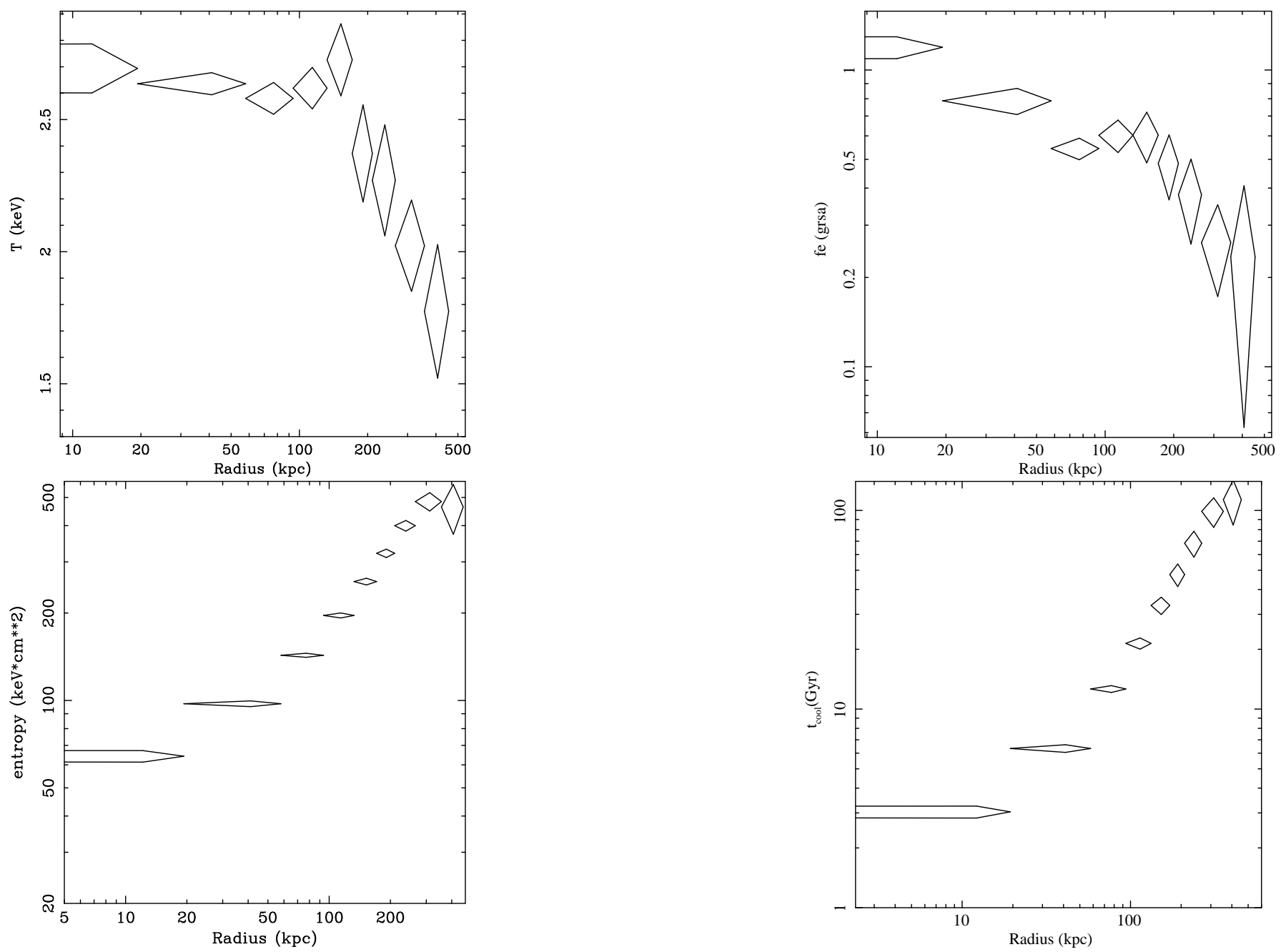

FIG. 2.- From left to right: the temperature profile of AWM 4 as obtained by the $X M M$ data, the abundance profile, the entropy profile and the cooling time profile.

$\chi^{2} /$ dof $=107 / 7$ for a single power law fit and $K_{0}=52 \pm 6$ $\mathrm{keV} \mathrm{cm}{ }^{2}, K_{100}=122 \pm 51 \mathrm{keV} \mathrm{cm}{ }^{2}$ and $\alpha=1.14 \pm 0.08$ with $\chi^{2} /$ dof $=7 / 6$ for a power law plus constant fit. The elevated central entropy of AWM 4 is reflected in the long central cooling time (calculated as $t_{\text {cool }}=5 / 2 \mathrm{kT} / \mathrm{n} \Lambda$, e.g., Peterson \& Fabian 2006, see lower right panel of Fig 2), $3.0 \pm 0.2 \mathrm{Gyr}$ in the inner bin. In this regard AWM 4 shares the same characteristics of the two radioquiet clusters, A 1650 and A 2244, of Donahue et al. (2005) and would be consistent with the hypothesis that a major past AGN outburst has completely erased the cool core. But the current $1.4 \mathrm{GHz}$ radio activity implies a recent (not much more than $\sim 10^{8}$ yr ago, the upper limit to the characteristic lifetime, i.e. synchrotron age, of the radio source) feeding of the central black hole, which is unlikely to come from the ambient hot gas which has an order of magnitude longer cooling time.

The anomalous nature of AWM 4, compared to the objects in the sample of Donahue et al. (2006), stands out clearly in a plot of central entropy versus $20 \mathrm{~cm}$ radio power (calculated as $\nu L_{\nu}$ where $L_{\nu}=4 \pi D_{L}^{2} S_{\nu}$, neglecting the K-correction term), as it can be seen in Fig 3 . We did not plot the two radio quiet objects of Donahue et al. (2005) because a homogeneous comparison with data at $1.4 \mathrm{GHz}$ is needed: for example a central radio source has been detected in A 1650 with a sensitive observation at $327 \mathrm{MHz}$ by Marković et al. (2004), but this can be explained as the old remnant of the previous very active AGN. On the contrary the $X M M$ data for AWM 4 suggests a surprising lack of low entropy gas surrounding an active and bright radio source at $1.4 \mathrm{GHz}$, although they cannot rule out its presence at scales smaller than the inner $20 \mathrm{kpc}$. For the clusters in the Donahue et al. (2006) sample, even though the entropy profiles are more detailed because of the higher spatial resolution of Chandra, they still rely on temperature determinations in inner bins with widths ranging from $7 \mathrm{kpc}$ (A 262, $\mathrm{z}=0.0163$ ) to $32 \mathrm{kpc}$ (PKS 0745-191, $\mathrm{z}=0.1028$ ) with 4 objects out of 9 with inner bins with width $\gtrsim 20 \mathrm{kpc}$.

\section{DISCUSSION}

Our investigation of current $X M M$ data shows no statistically significant surface brightness or spectral variations in the core at a level greater than $1-2 \sigma$ (as it is also the case for the temperature variations quoted in O'Sullivan et al. 2005, see their table 5). In this section we will discuss possible scenarios to explain the characteristics of this peculiar source.

There is a clear observational dichotomy between cool core (CC), relaxed clusters and non-cool core (NCC), disturbed clusters. This is not only based on the cen- 


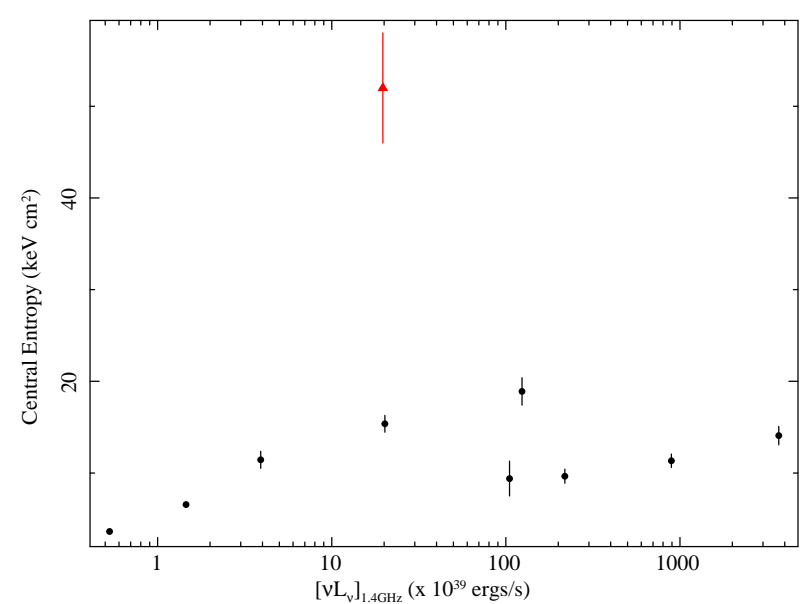

FIG. 3. - Central entropy values taken from the sample of Donahue et al. (2006) (black circles) and for AWM 4 (red triangle). The radio power $\nu L_{\nu}$ at $20 \mathrm{~cm}$ is taken from the NVSS (Condon et al. 1998).

tral temperature gradient or central cooling time, but also for example on the degree of regularity of morphologies (as measured by power ratios, which strongly correlates with the strength of the cooling, as measured for local and moderate redshift clusters: Buote \& Tsai 1996; Bauer et al. 2005) or the presence/absence of a central abundance gradient (De Grandi \& Molendi 2001). A possible new class of objects can include systems like A 1650 and A 2244, which have all the characteristics of relaxed objects but lack a cool core due to a strong episode of AGN feedback. Regardless of this possible last class, it is commonly assumed that mergers can transform CC clusters into NCC ones, depending on factors such as the initial strength of the cooling core, how off-center the merger is and the mass-ratio of the merging system (McGlvnn \& Fabian 1984; Gómez et al. 2002; Ritchie \& Thomas 2002). There is still debate and controversial results from simulations regarding the effectiveness of mergers in disrupting cool cores and being responsible for the $\mathrm{CC} / \mathrm{NCC}$ dichotomy (Poole et al. 2006; Burns et al. 2007; McCarthy et al. 2007). The inability of current hydro-dynamical simulations in reproducing realistic cool cores may be one of the reason for these controversial results. Nevertheless, it is crucial to discuss a merger hypothesis for the properties of AWM 4. The central radio source has some similarities with a wide-angle-tail (WAT) source, which it has been argued can be shaped by ICM ram pressure produced in cluster mergers (e.g., Burns et al. 1994; Gomez et al. 1997). This is a well motivated hypothesis for some of the most relevant cases like the ones presented in Gomez et al. (1997) which present clear evidence of disturbed X-ray morphologies, but it can not hold in cases like the one of AWM 4. Small scale disturbances due to accretion of small mass objects, like the simulations described in Ascasibar \& Markevitch (2006) can reproduce the characteristic X-ray spiral structure seen for example in the core of Perseus and provide the necessary relative ICM motions to explain the WAT-like jet bending. Alternatively jet bending can result from instabilities, preexisting radio plasma, as discussed for Hydra A (Nulsen et al. 2005), or by jet precession, as in the spectacular case of the cluster RBS797 (Gitti et al. 2006). A good comparison can be made with the recent Chandra observation of the prototypical WAT host cluster A 1446 (Douglass et al. 2007): in this cluster there is a complex $\mathrm{X}$-ray morphology in the inner $200 \mathrm{kpc}$ and there is a flat abundance profile, a sensitive indicator of an ongoing merger. AWM 4 has very different properties in the $\mathrm{X}$-rays and in the optical, in particular its fossil property would be difficult to understand in a recent major merger scenario. The same conclusion has been reached by O'Sullivan et al. (2005).

Clearly given the spatial resolution of the $X M M$ data, the possibility of the accretion of a low-mass object, like a small group, a cool galaxy corona, able to survive stripping and thermal evaporation (Sun et al. 2007), or even a gas rich dwarf galaxy, can not be ruled out as the cause of cooled gas that triggered the radio jet in AWM 4 .

It is also useful to compare AWM 4 with the suggested evolutionary scenario discussed in Buote et al. (2005). Systems like A 644 are just settling down from a major merger to establish the initial cooling/feedback loop and there is no radio emission until the core is basically relaxed. A possible interpretation is that AWM 4 would instead have represented the final stage of the cycle of AGN feedback, like A 1650 and A 2244, if it were not for a small-merger/accretion related event. The limits posed by the $X M M$ image to the center offset is $8 \pm 2$ $\mathrm{kpc}$, but this determination is hampered by low $X M M$ spatial resolution (the PSF FWHM on-axis corresponds to $2 \mathrm{kpc}$ at the redshift of the source).

It is still possible to reason along the lines of the model for example of Voit \& Donahue (2005) but we have to allow the previous AGN outflow of $\sim 10^{46} \mathrm{ergs} \mathrm{s}^{-1}$ (capable of raising the central entropy to the observed $\sim 50$ $\mathrm{keV} \mathrm{cm}^{2}$ ) of having been not able to completely stop cooling near the very core, much alike the cool coronae observed in NGC 3842 (in A 1367) and NGC 4874 (in Coma) by Sun et al. (2005). In these sources if the AGN mechanical power, associated with the observed radio emission, has the same magnitude observed in cool core clusters, it would have completely disrupted the observed X-ray soft coronae. Remaining cooling gas (or gas supplied by stellar mass loss) could have triggered the current outburst: assuming the energy released by accretion onto a black hole to be $\eta \dot{M} c^{2}$ with $\eta=0.1$ we have $\dot{M}=0.0017 L_{X, 43} M_{\odot} \mathrm{yr}^{-1}$, where $L_{X, 43}=10^{43}$ erg s${ }^{-1}$; an accretion rate as low as $0.017 M_{\odot} \mathrm{yr}^{-1}$ could sustain a total mechanical luminosity of $10^{44} \mathrm{erg} \mathrm{s}^{-1}$, a thousand times higher than the radio luminosity.

Measurements of X-ray cavity sizes and surrounding gas pressure have provided unique estimates of the ratio of jet power to synchrotron power. The jet power determined from X-ray cavity data shows a clear correlation with synchrotron power (core plus lobes); the median ratio of jet (cavity) power to synchrotron power is $\sim 100$, with mean $\sim 2800$ owing to large (ranging from a few to a few thousand) scatter (Bîrzan et al. 2004; McNamara \& Nulsen 2007, in particular their Fig.7); this is interestingly on the high side of theoretical expectations (e.g., De Young 1993). Given its current radio luminosity the central radio source in AWM 4 can well provide up to $10^{43}-10^{44} \mathrm{erg} \mathrm{s}^{-1}$ of mechanical energy into the ICM. These luminosities would be sufficient to carve $\mathrm{X}$-ray cavities in the ICM: if for example the mechani- 
cal luminosity associated with the radio jets in AWM4 is $10^{43} \mathrm{erg} \mathrm{s}^{-1}$ and assuming an enthalpy of $4 p V$, appropriate for cavities dominated by relativistic particles, and a duration of the outburst of $10^{7}$ years, the energy created would result in a pair of spherical cavities with radius $\approx 3.5 \mathrm{kpc}$ located within $\approx 20 \mathrm{kpc}$ from the center, perhaps coincident with the inner bright radio "knots" at $21^{\prime \prime}(13 \mathrm{kpc})$ and $34^{\prime \prime}(21 \mathrm{kpc})$ from the center. Cavities of these sizes would produce a level of contrast in surface brightness above $30 \%$, which is the contrast of evident bubbles like the ones in A 2052 and Hydra A. If cavities are detected in AWM 4, it would establish that AGN mechanical heating has taken place in the past $\sim 10^{8} \mathrm{yr}$, given the fact that any cavities that might be seen today may only have come from the currently visible radio jet: for example the buoyancy time scale for a bubble of 3.5 $\mathrm{kpc}$ at $\mathrm{R}=21 \mathrm{kpc}$ is $9 \pm 2 \times 10^{7} \mathrm{yr}$, calculating the gravitational acceleration as $g=G M(<R) / R^{2}$ using the mass profile derived in Gastaldello et al. (2007); using instead $g \sim 2 \sigma^{2} / \mathrm{R}$ where $\sigma=243 \pm 24 \mathrm{~km} / \mathrm{s}$ (Davies et al. 1987) is the stellar velocity dispersion of the central galaxy would result in $8 \pm 2 \times 10^{7} \mathrm{yr}$.

Another indication of the energy of the previous outburst which has disrupted the cool core of AWM 4 comes from the analysis of Rebusco et al. (2006). By comparing the observed peaked iron abundance profile for a sample of groups and clusters, including Perseus, with the prediction of a simple model invoking the metal ejection from the BCG and the subsequent diffusion of metals by stochastic gas motions, they found for AWM 4 the largest value of the diffusion coefficient, implying the strongest level of gas mixing.

\section{CONCLUSIONS}

AWM 4 provides an early critical test to the new born theory of AGN feedback, and it exposes our poor understanding of the details of the fueling and triggering of AGN outbursts, including the part, if any, played by mergers, as for example highlighted in the future issues to be addressed by McNamara \& Nulsen (2007). We must understand the new cycle of heating and cooling of a relaxed galaxy cluster, as depicted for example in the AGN feedback loop by Tucker et al. (2007), and its connection to the pre-Chandra loop of mergers and relaxation in cool cores (e.g., Buote 2002).

We would like to thank S. Ettori, M. Gitti, P.J. Humphrey, D. Pierini, S. Zibetti and the anonymous referee for useful comments. F.G. and D.A.B. gratefully acknowledge partial support from NASA grant NAG513059, issued through the Office of Space Science Astrophysics Data Program. This work is based on observations obtained with $X M M$ an ESA science mission with instruments and contributions directly funded by ESA member states and the USA (NASA).

\section{REFERENCES}

Ascasibar, Y. \& Markevitch, M. 2006, ApJ, 650, 102

Bauer, F. E., et al. 2005, MNRAS, 359, 1481

Binney, J. 2005, Royal Society of London Philosophical Transactions Series A, 363, 739

Bîrzan, L., et al. 2004, ApJ, 607, 800

Buote, D. A. 2002, in Astrophysics and Space Science Library,

Vol. 272, Merging Processes in Galaxy Clusters, ed. L. Feretti,

I. M. Gioia, \& G. Giovannini, 79-107

Buote, D. A., et al. 2005, ApJ, 630, 750

Buote, D. A. \& Tsai, J. C. 1996, ApJ, 458, 27

Burns, J. O. 1990, AJ, 99, 14

Burns, J. O., et al. 2007, ApJ accepted, arXiv:0708.1954

Burns, J. O., et al. 1994, ApJ, 423, 94

Churazov, E., et al. 2002, MNRAS, 332, 729

Condon, J. J., et al. 1998, AJ, 115, 1693

Davies, R. L., et al. 1987, ApJS, 64, 581

De Grandi, S., et al. 2004, A\&A, 419, 7

De Grandi, S. \& Molendi, S. 2001, ApJ, 551, 153

De Young, D. S. 1993, ApJ, 405, L13

Donahue, M., et al. 2006, ApJ, 643, 730

Donahue, M. \& Voit, G. M. 2004, in Clusters of Galaxies: Probes of Cosmological Structure and Galaxy Evolution, ed. J. S.

Mulchaey, A. Dressler, \& A. Oemler, 143

Donahue, M., et al. 2005, ApJ, 630, L13

Douglass, E. M., et al. 2007, ApJ accepted, arXiv:0706.2519

Dunn, R. J. H. \& Fabian, A. C. 2006, MNRAS, 373, 959

Forman, W., et al. 2005, ApJ, 635, 894

Gastaldello, F., et al. 2007, ApJ, 669, 158

Girardi, M., et al. 1997, ApJ, 490, 56

Gitti, M., et al. 2006, A\&A, 448, 853

Gómez, P. L., et al. 2002, ApJ, 569, 122

Gomez, P. L., et al. 1997, ApJ, 474, 580

Grevesse, N. \& Sauval, A. J. 1998, Space Science Reviews, 85, 161
Jones, L. R., et al. 2003, MNRAS, 343, 627

Koranyi, D. M. \& Geller, M. J. 2002, AJ, 123, 100

Markevitch, M., et al. 2000, ApJ, 541, 542

Marković, T., et al. 2004, in The Riddle of Cooling Flows in Galaxies and Clusters of galaxies, ed. T. Reiprich, J. Kempner, \& N. Soker, 61

McCarthy, I. G., et al. 2007, ArXiv e-prints, arXiv:0706.2768

McGlynn, T. A. \& Fabian, A. C. 1984, MNRAS, 208, 709

McNamara, B. R. \& Nulsen, P. 2007, Annual Review of

Astronomy and Astrophysics, 45

McNamara, B. R., et al. 2005, Nature, 433, 45

Motl, P. M., et al. 2004, ApJ, 606, 635

Narayan, R. \& Medvedev, M. V. 2001, ApJ, 562, L129

Neumann, M., et al. 1994, A\&AS, 106, 303

Nulsen, P. E. J., et al. 2006, ArXiv Astrophysics e-prints, arXiv:astro-ph/0611136

Nulsen, P. E. J., et al. 2005, ApJ, 628, 629

O'Sullivan, E., et al. 2005, MNRAS, 357, 1134

Peres, C. B., et al. 1998, MNRAS, 298, 416

Peterson, J. R. \& Fabian, A. C. 2006, Phys. Rep., 427, 1

Poole, G. B., et al. 2006, MNRAS, 373, 881

Rebusco, P., et al. 2006, MNRAS, 372, 1840

Ritchie, B. W. \& Thomas, P. A. 2002, MNRAS, 329, 675

Scheuer, P. A. G. 1974, MNRAS, 166, 513

Sun, M., et al. 2007, ApJ, 657, 197

Sun, M., et al. 2005, ApJ, 619, 169

Tabor, G. \& Binney, J. 1993, MNRAS, 263, 323

Tucker, W., et al. 2007, Scientific American, 296, 42

Voit, G. M. \& Donahue, M. 2005, ApJ, 634, 955

White, R. L., et al. 1997, ApJ, 475, 479 